\title{
Insight into unique somitogenesis of yak (Bos grunniens) with one additional thoracic vertebra
}

\author{
Yu Wang ${ }^{1}$, Haoyang $\mathrm{Cai}^{2}$, Xiaolin Luo ${ }^{3}, \mathrm{Yi} \mathrm{Ai}^{4}$, Mingfeng Jiang ${ }^{1^{*}}$ (D) and Yongli Wen ${ }^{4^{*}}$
}

\begin{abstract}
Background: The yak is a species of livestock which is crucial for local communities of the Qinghai-Tibet Plateau and adjacent regions and naturally owns one more thoracic vertebra than cattle. Recently, a sub-population of yak termed as the Jinchuan yak has been identified with over half its members own a thoracolumbar vertebral formula of T15L5 instead of the natural T14L5 arrangement. The novel T15L5 positioning is a preferred genetic trait leading to enhanced meat and milk production. Selective breeding of this trait would have great agricultural value and exploration of the molecular mechanisms underlying this trait would both accelerate this process and provide us insight into the development and regulation of somitogenesis.
\end{abstract}

Results: Here we investigated the genetic background of the Jinchuan yak through resequencing fifteen individuals, comprising five T15L5 individuals and ten T14L5 individuals with an average sequencing depth of $>$ 10X, whose thoracolumbar vertebral formulae were confirmed by anatomical observation. Principal component analysis, linkage disequilibrium analysis, phylogenetic analysis, and selective sweep analysis were carried out to explore Jinchuan yak's genetic background. Three hundred and thirty candidate markers were identified as associated with the additional thoracic vertebrae and target sequencing was used to validate seven carefully selected markers in an additional 51 Jinchuan yaks. The accuracies of predicting 15 thoracic vertebrae and 20 thoracolumbar vertebrae with these 7 markers were 100.00 and 33.33\% despite they both could only represent 20\% of all possible genetic diversity. Two genes, PPP2R2B and TBLR1, were found to harbour the most candidate markers associated with the trait and likely contribute to the unique somitic number and identity according to their reported roles in the mechanism of somitogenesis.

Conclusions: Our findings provide a clear depiction of the Jinchuan yak's genetic background and a solid foundation for marker-assistant selection. Further exploitation of this unique population and trait could be promoted with the aid of our genomic resource.

Keywords: The Jinchuan yak, Somitogenesis, Population genetics, Plateau adaptation, Molecular breeding, Markerassisted selection

\section{Background}

The yak is a species of livestock that is well-adapted to the extreme environment of plateaus and plays an important role in local residents' lives. Recently, a unique sub-population was identified and referred to as the

\footnotetext{
* Correspondence: mingfengjiang@vip.sina.com; 66550344@qq.com ${ }^{1}$ College of Life Science and Technology, Southwest Minzu University, Chengdu 610041, Sichuan, China

${ }^{4}$ Key Laboratory of Sichuan Province for Qinghai-Tibetan Plateau Animal Genetic Resource Reservation and Exploitation, Chengdu 610041, China Full list of author information is available at the end of the article
}

Jinchuan yak due to its higher meat yield owing to an additional thoracic vertebra [1]. For example, the average net weight of male Jinchuan yaks with 15 pairs of ribs could be over $12 \mathrm{~kg}$ heavier than that of male Jinchuan yaks with 14 pairs of ribs [2], demonstrating the great economic potential of the T15L5 Jinchuan yak. The normal thoracolumbar vertebral formula of yak is T14L5 (14 thoracic vertebrae and 5 lumbar vertebrae). However, several alternative formulae exist for the Jinchuan yak. It was reported that around $52 \%$ of Jinchuan yaks are $\mathrm{T} 15 \mathrm{~L} 5,37 \%$ are $\mathrm{T} 14 \mathrm{~L} 5,8 \%$ are $\mathrm{T} 14 \mathrm{~L} 6$, and $3 \%$ are

(c) The Author(s). 2020 Open Access This article is distributed under the terms of the Creative Commons Attribution 4.0 International License (http://creativecommons.org/licenses/by/4.0/), which permits unrestricted use, distribution, and 
T15L4 which were determined by anatomical observation [3]. The same number of thoracic vertebrae but different number of lumbar vertebrae increases the genetic heterogeneity among individuals with 15 ribs. Unfortunately, the thoracolumbar vertebral formula is often determined post-slaughter as it is hard to judge whether a live yak expresses an extra vertebra. Therefore, there is an urgent need to find a method to select individuals with more vertebrae.

A mature somite, which consists of two compartments: the sclerotome and the dermomyotome, is derived from the presomitic mesoderm (PSM) while vertebrae are derived from the sclerotome compartment [4]. The number of somites is controlled by a segmentation clock [5] and the identity of somites (vertebrae) is specified by Hox genes in the PSM [6]. A faster segmentation clock could produce embryos with increased vertebral number [7]. Substantial advances have been made in the molecular mechanisms of embryonic somitogenesis, which strongly support the investigation of a wide variety of phenotypic traits relevant to vertebrae [8].

The increased vertebral number has not only been observed in yaks, but also in cattle [9], sheep [10] and certain breeds of pigs [11-13]. Studies on multi-rib pigs date back to the early twentieth century [14]. In recent years, the methods researchers commonly take to locate regions and loci that impact the number of vertebrae include quantitative trait loci (QTL) analysis $[15,16]$ and genome-wide association study (GWAS) [11, 12], which require large numbers of (around one thousand) offspring derived from a few (around four) parents. One gene with considerable influence on thoracic vertebral number (TVN) in pigs was found to be Vertnin (VRTN) [11]. The biological mechanism of VRTN affecting somitogenesis has been reported to be the acceleration of the segmentation clock through the Notch signaling pathway [17].

Besides increased vertebral number, the Jinchuan yak also possesses other advantageous characteristics including high milk production [2] and an earlier age at first calving $(85.4 \%$ of the Jinchuan yak calved first at three years old, which is one year earlier than average) [18], so one of the aims of this study is to dissect the population genetic characteristics of the Jinchuan yak via comparing with the Qinghai-plateau yak. Another important aim of our study is to seek markers which can be used to selectively breed Jinchuan yaks with a thoracolumbar vertebral formula T15L5. We also seek a greater understanding of the mechanisms relevant to the aberrant somitogenesis. To this end, we performed whole genome resequencing of 15 yaks whose thoracolumbar vertebral formulae were confirmed by an anatomical method to exclude the genetic heterogeneity introduced by the T15L4 and T14L6 individuals.

\section{Results}

Reads statistics and variants annotation Sequencing and variants calling

A total of $463.64 \mathrm{G}$ bases composed of 1545 million pairs of raw reads were generated on an Illumina HiSeq $\mathrm{X}$ Ten sequencing platform. 1522 million pairs of clean reads containing $456.61 \mathrm{G}$ bases remained after filtering (Table 1). The average sequencing depths of samples reached above 10X, which ensured the accuracy of the genotypes calling. Around 17 million SNPs and 2 million InDels were called with GATK. The average variants calling rate for all individuals was $93.4 \%$ (94.3\% for SNPs and $86.4 \%$ for InDels).

\section{Population-level genotypes and variants}

As the aim of this study was to discover population-level features rather than the characteristics of any one individual, population-level genotypes were identified first. Population-level genotypes were defined as the genotypes those were the same in all five individuals in one population. Ten million out of 17 million SNPs were identified to be population-level variants in at least one population and the relationship between loci harboring these variants is shown in Fig. 1. We observed the number of loci shared between the ZC population (five Jinchuan yaks whose thoracolumbar vertebral formula was T14L5) and YC population (five Jinchuan yaks whose thoracolumbar vertebral formula was T15L5) $(1,612,871)$ was less than the number of loci they shared with the GY population (five Qinghai-plateau yaks whose thoracolumbar vertebral formula was T14L5) (ZC-GY: 1,980,530 and YC-GY: 1,831, 695). The variants of each population that resided in population-level loci were further functionally annotated

Table 1 Summary of each individuals' sequencing information

\begin{tabular}{lllll}
\hline Samples & \# Raw reads & \# Clean reads & Clean Base (Gb) & Average Depth \\
\hline YC1 & $106,652,787$ & $105,113,768$ & $31,534,130,400$ & 11.94 \\
YC2 & $127,528,345$ & $126,155,996$ & $37,846,798,800$ & 14.34 \\
YC3 & $110,054,170$ & $109,046,964$ & $32,714,089,200$ & 12.39 \\
YC4 & $98,439,661$ & $97,186,917$ & $29,156,075,100$ & 11.04 \\
YC5 & $120,316,613$ & $118,876,788$ & $35,663,036,400$ & 13.51 \\
ZC1 & $93,795,354$ & $92,871,350$ & $27,861,405,000$ & 10.55 \\
ZC2 & $89,386,096$ & $81,346,718$ & $24,404,015,400$ & 9.24 \\
ZC3 & $98,237,486$ & $97,483,485$ & $29,245,045,500$ & 11.08 \\
ZC4 & $94,029,951$ & $93,143,964$ & $27,943,189,200$ & 10.58 \\
ZC5 & $94,768,929$ & $93,561,321$ & $28,068,396,300$ & 10.63 \\
GY1 & $120,612,083$ & $118,972,444$ & $35,691,733,200$ & 13.52 \\
GY2 & $106,477,289$ & $105,676,535$ & $31,702,960,500$ & 12.01 \\
GY3 & $93,962,434$ & $93,240,641$ & $27,972,192,300$ & 10.60 \\
GY4 & $93,469,905$ & $92,595,105$ & $27,778,531,500$ & 10.52 \\
GY5 & $97,742,066$ & $96,765,431$ & $29,029,629,300$ & 11.00 \\
\hline
\end{tabular}




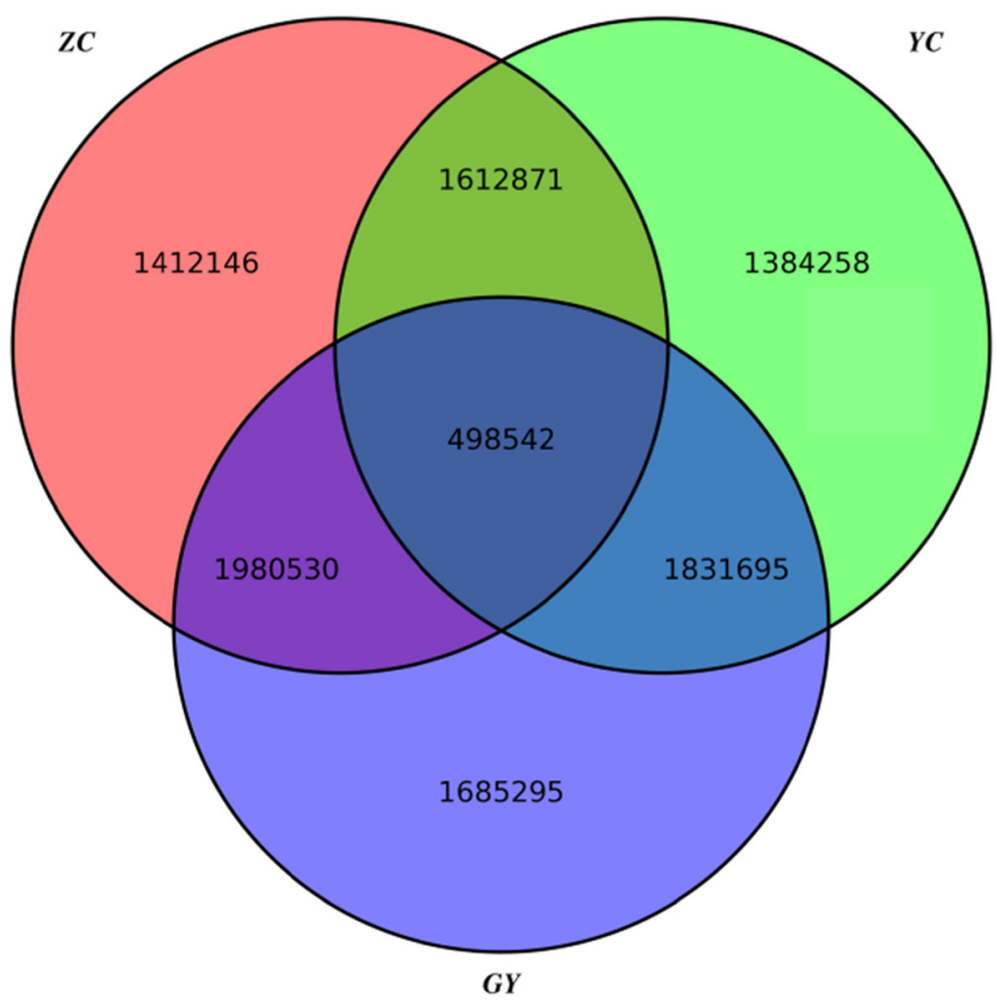

Fig. 1 Shared loci harboring population-level genotypes among populations

(Table 2). The number of variants found in each type was similar in the three populations. The variants in exonic regions of genes hit around 5 thousand genes per population (ZC: 4670; YC: 4943; GY: 4780) and the nonsynonymous SNVs hit around 3 thousand genes per population (ZC: 3040; YC: 3257; GY: 3100). A stopgain SNV (gene14456: rna17636:exon2:c.G167A:p.W56X) and a nonsynonymous SNV (gene14456:rna17636:exon3:c.G1661C:p.R554P) were observed in the gene VRTN, which was reported to affect the somite number in pigs $[11,17]$. These two SNVs were present in all individuals with an average $8 \mathrm{X}$ sequencing depth. A stoploss SNV (gene20267:rna24673:exon6: c.A365G:p.X122W) and a nonsynonymous SNV (gene20267:rna24673:exon6:c.C295A:p.R99S) were observed in the gene TMEM200 in all individuals with an average $>12 \mathrm{X}$ sequencing depth. Finally, another transmembrane protein TMEM192 (discussed in the section Association analysis) was also identified because nine population-level variants associated with the trait resided within $5 \mathrm{~kb}$ of the gene.

\section{Population genetics analysis Principal component analysis}

Fifteen individuals were divided into two groups (5 Qinghai-plateau yaks in one group and 10 Jinchuan yaks in another group) by the principle component 1 (PC1) (Fig. 2). The PC1 is the principal component with the
Table 2 Annotation of population-level SNV of each population

\begin{tabular}{llll}
\hline Values & ZC & YC & GY \\
\hline splicing & 93 & 105 & 96 \\
intronic & 228,602 & 239,609 & 228,036 \\
intergenic & 668,240 & 694,364 & 655,789 \\
UTR3 & 3488 & 3662 & 3411 \\
UTR5 & 1050 & 1131 & 1071 \\
ncRNA_exonic & 1230 & 1253 & 1168 \\
ncRNA_splicing & 1 & 2 & 1 \\
ncRNA_intronic & 2662 & 2628 & 2636 \\
ncRNA_UTR5 & 2 & 3 & 3 \\
upstream & 8342 & 9110 & 8458 \\
downstream & 8360 & 8948 & 7798 \\
upstream;downstream & 221 & 270 & 245 \\
exonic & 13,084 & 14,769 & 13,097 \\
exonic;splicing & 18 & 18 & 19 \\
nonsynonymous SNV & 6451 & 7307 & 6526 \\
synonymous SNV & 4723 & 5266 & 4712 \\
stopgain SNV & 61 & 76 & 62 \\
stoploss SNV & 14 & 12 & 13 \\
unknown & 1853 & 2126 & 1803 \\
\hline
\end{tabular}




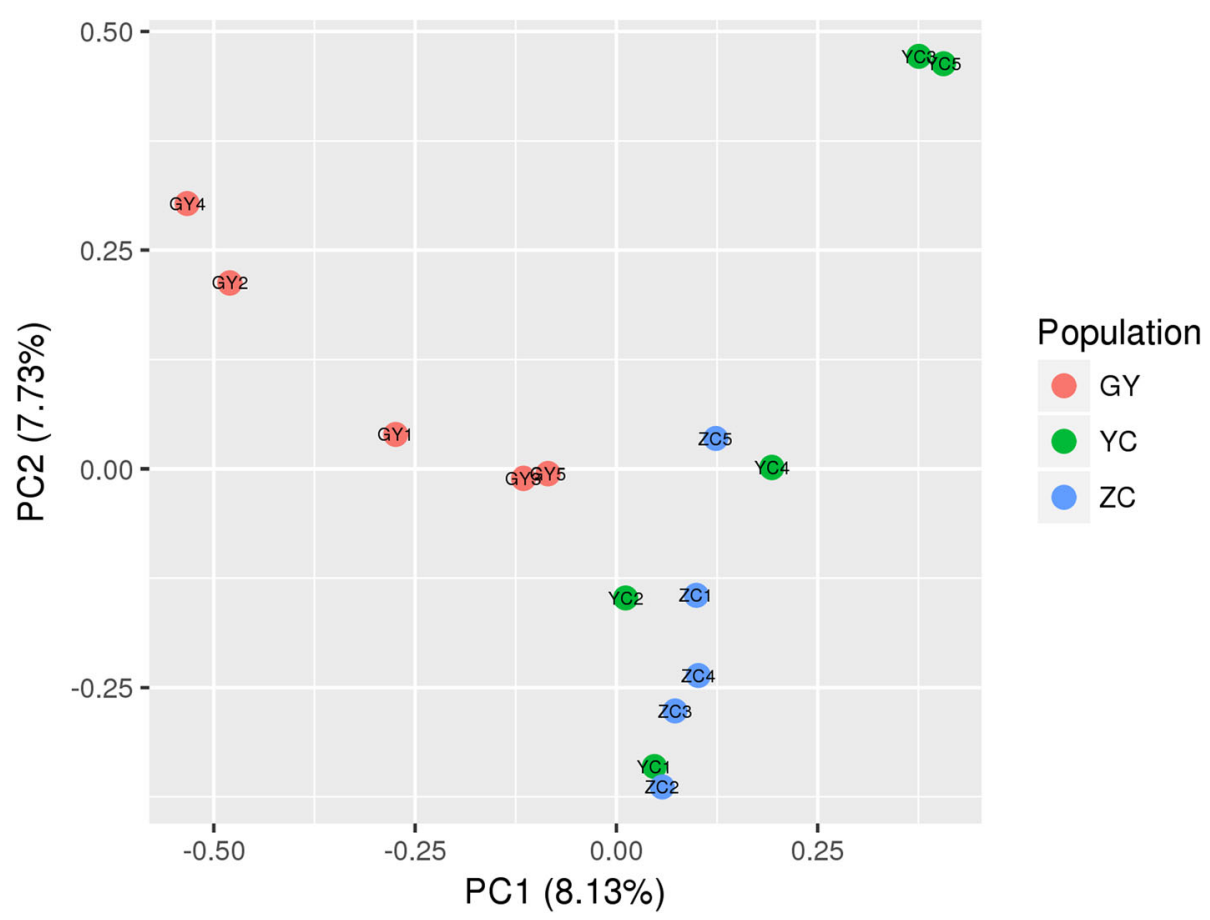

Fig. 2 Principal component analysis

greatest variance; hence this indicated that the genetic difference between the Jinchuan yak and the Qinghaiplateau yak was the greatest and the result was in line with a general expectation. However, we should also note that: (1) the proportion of the information contained in the $\mathrm{PC} 1(8.13 \%)$ and the $\mathrm{PC} 2(7.73 \%)$ was very close; (2) the division of individuals according to the PC2 was different from that according to the PC1. It reflected the genetic variance that the $\mathrm{PC} 2$ represented accounted for a large proportion and was different from what the PC1 represented. Interestingly, the manner that PC2 separated individuals was nearly the same as our phenotypic grouping manner, which suggested the genetic variance represented by the PC2 might overlap with the genetic variance impacting the thoracolumbar vertebral formula. As no principle component separated individuals into two groups corresponding to the $\mathrm{YC}$ population and the $\mathrm{ZC}$ population well, the region regulating the trait was putatively a small proportion of genomic regions.

\section{Linkage disequilibrium}

The slowest decay rate and the highest level of linkage disequilibrium (LD) were observed in the YC population, whereas the rapidest decay rate and the lowest level of LD were observed in the GY population (Fig. 3).

This phenomenon can be attributed to the intense artificial selection for the extra thoracic vertebra trait in heavier yaks which may lead to the decline in genetic diversity and increased linkage among loci. This was also likely reflected in the effective population size $\left(N_{e}\right)$ for the YC population which was the smallest compared with the ZC and GY populations. Due to the slow LD decay rate of the YC population, it became less difficult to select loci linked to the causal variants determining the trait of increased vertebral number for selective breeding.

\section{Phylogenetic tree}

The NJ (neighbor-joining) tree split individuals into three groups, which are highlighted with blocks of different colors (Fig. 4). It was reasonable to sort five Qinghai-plateau yaks into one group (highlighted with a red block) because these individuals have lived in an environment that is different from Jinchuan yak's for a long time.

However, the large genetic distance within Jinchuan yak suggested a significant genetic diversity exists in the Jinchuan yaks. Considering the genetic distance between Jinchuan yaks, Qinghai-plateau yaks and the outgroup (cattle), it suggested that Jinchuan yaks could be further divided into two subpopulations, which were highlighted with a green block and a yellow block. The genetic distance from the green block to the red block was closer than to the yellow block. And the yellow block was closer to the outgroup cattle than any other blocks were.

In light of the previously reported phylogeographical study of domestic and wild yaks based on complete mitochondrial sequences [19], it was suggested that the 


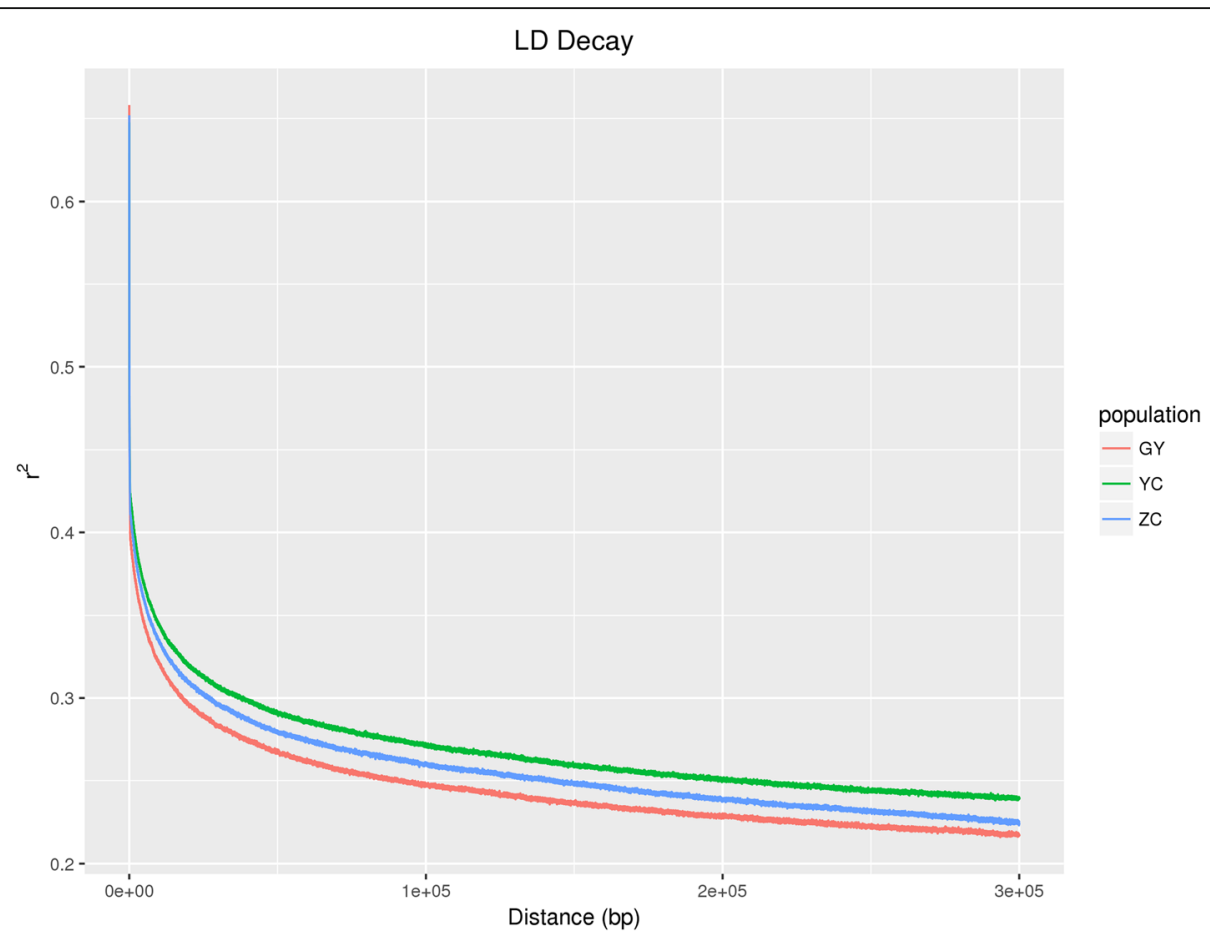

Fig. 3 Decay of linkage disequilibrium

animals highlighted in the yellow block were from one lineage (referred to as lineage 2 to make the nomenclature to be consistent with the previous research [19], the same below) because of its closer proximity to the outgroup cattle, and those highlighted in the green block were from another lineage (referred to as lineage 1, the individuals constitute this lineage are widely distributed around Qinghai-Tibetan Plateau) due to its closer proximity to the red block than the yellow block. The numbering of the lineages here was consistent with the nomenclature used in the phylogeographical study [19], which concluded that three different lineages evolved allopatrically and then reunited into one gene pool before the start of domestication. Four individuals from ZC
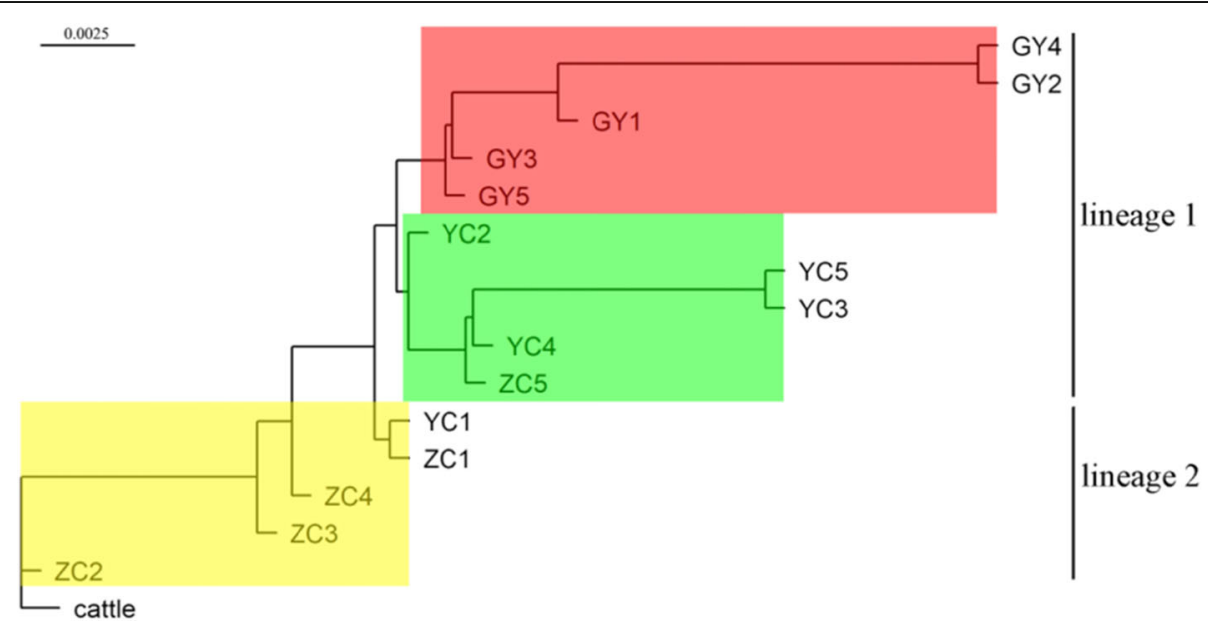

Fig. 4 Neighbor-join tree constructed using (1-IBS) distance between animals. Cattle was used as an outgroup. Those with close (1-IBS) distances were highlighted with a block with the same color. Of note, the bar of the plot only represented distances between the nodes except tips. The unit distance from a tip to its corresponding closest node was different from the bar. The distance of the cattle's tip to its corresponding closest node was 0.33 and the average distance of all other yaks' tips to their corresponding closest nodes was 0.11 . The purpose of adjusting the unit distance was to better display the distance between individuals and blocks. The lineage to which each animal belongs was labeled on the right of the tree 
population were from lineage 2. And four individuals from YC population and all five individuals from GY population were from lineage 1 . This suggests that variants determining the trait were inherited from a common ancestor of lineage 1 and were further fixed in $\mathrm{YC}$ population, which resulted in $52 \%$ of Jinchuan yak with one additional thoracic vertebra [3]. Therefore, the markers we identified here may still be useful to select those originating from common ancestors in other places.

Another interesting observation was that the genetic distances between individuals shown in the NJ tree were similar to the distances between individuals reflected by PCA (Fig. 2), such as the close distances between GY2 and GY4 and between YC3 and YC5. However, the relationship between $\mathrm{ZC} 1$ and $\mathrm{YC} 1$ presented in the PCA was not as close as it was in the NJ tree. These differences are reasonable to observe because the (1-IBS) distance matrix was calculated using 14 million whole genome SNVs, but the variances of $\mathrm{PC} 1$ and $\mathrm{PC} 2$ only contributed $15.86 \%$ to the total variance. We also observed that the relationships presented in the NJ tree were closer to what PC2 reflected, where 5 individuals from the GY population and 3 individuals from YC populations were located above the $\mathrm{y}=0$ line and 4 individuals from the ZC population located below the $y=0$ line. In other words, $\mathrm{PC} 2$ grouped individuals from the GY and YC populations into a cluster and individuals from the ZC population into another cluster which was consistent with the result of the NJ tree. This suggests that the similarity inherited from the same ancestors was second only to the similarity that gradually accumulated through living in the same environment. In summary, the results of PCA and phylogenetic analysis indicated that two lineages existed in our samples, which contrasted with their classification based on the differences in morphologies and geographical distribution.

\section{Regions under selective pressure}

The nucleotide diversity (pi) and populationdifferentiation statistic (Fst) were calculated using whole genome SNVs. The average pi of each population (YC: 0.00134, ZC: 0.00130, GY: 0.00123) was similar to other domestic yaks' pi that was also calculated from whole genome sequencing data (unselected landraces (D2): 0.00138, Tianzhu white yaks (D1): 0.00137, 36]. The average Fst between Jinchuan yaks and Qinghai-plateau yaks was 0.0415 ; this was larger than the Fst $(0.0213)$ between D1 and D2 but smaller than that $(0.0582)$ between D1 + D2 and wild yaks [20], which indicated that a relatively high population differentiation exists between the Plateau yak (represented by Qinghai-plateau yaks) and the Valley yak (represented by Jinchuan yaks). The pattern identified in our study was similar to that found by others using the same type of data which indicated that our data truly reflected the genetic characteristics of the objects we selected.

To explore the source of the generation of an extra vertebra and advantageous productive traits of Jinchuan yaks from an evolutionary perspective, selective sweep analysis was conducted between Jinchuan yaks and Qinghai-plateau yaks. Qinghai-plateau yaks were chosen as the control group because they belong to Plateau yak and Jinchuan yaks belong to Valley yak. The genetic distance between them should be relatively far away. The 5 $\mathrm{kb}$ regions whose Fst and pi log-ratio were both in the top $5 \%$ were regarded as regions under strong selective sweeps. 1393 windows covering $30.8 \mathrm{Mb}$ genomic regions and 4154 windows covering $62.5 \mathrm{Mb}$ genomic regions were chosen for the GY population (which refers to 5 Qinghai-plateau yaks) and the JC population (which refers to 10 Jinchuan yaks), respectively. The size of the regions under selective sweep in the Qinghai-plateau yak was less than half that of the Jinchuan yak. A possible reason for this phenomenon was that the habitat of the Qinghai-plateau yak seldom changed but the habitat of the Jinchuan yak might have undergone a major change. The genes that overlapped with these regions (S1 Table) were identified as the candidate genes under strong selective sweep. Furthermore, six genes (DMD, GPC6, KLF12, MAGI2, NXPH1, and TTC13) were identified in both populations because they overlapped with different windows having different pi log-ratio. The regions overlapping with genes are represented as green points in Fig. 5. A notable phenomenon revealed by our data was that the green points were generally located more internally relative to the blue points, which didn't overlap with genes but were under stronger selective sweep than the green points were. It suggested that the regions overlapping with genes were more stable than the regions that were likely to be functionless.

The genes under strong selective sweep were used to perform enrichment analysis in DAVID for the JC and GY populations. The enriched terms are listed in Table 3. Among the genes under selective sweep for the JC population, 27 genes (COL4A6, COL2A1, COL12A1, MEP1A besides genes whose name beginning with 'LOC') were involved in the 'protein digestion and absorption pathway', 14 genes (ERBB4, ADCY3, ADCY1, P2RX1, PDE1C, CCKBR, HRH1, PLCB1, ADRA1A, RYR2, ATP2A3, GRM1, LOC102275229, LOC102287375) were located in the 'calcium signaling pathway', 10 genes (SLC1A7, SLC38A1, PLCB1, ADCY3, ADCY1, GRM1, GRM8, GRIK3, GRIA3, GRIA4) resided in the 'glutamatergic synapse pathway'. In short, pathways related to two types of functions were significantly enriched: one was related to energy ('protein digestion and absorption', 'alpha-linolenic acid metabolism' and 'pancreatic secretion') and 


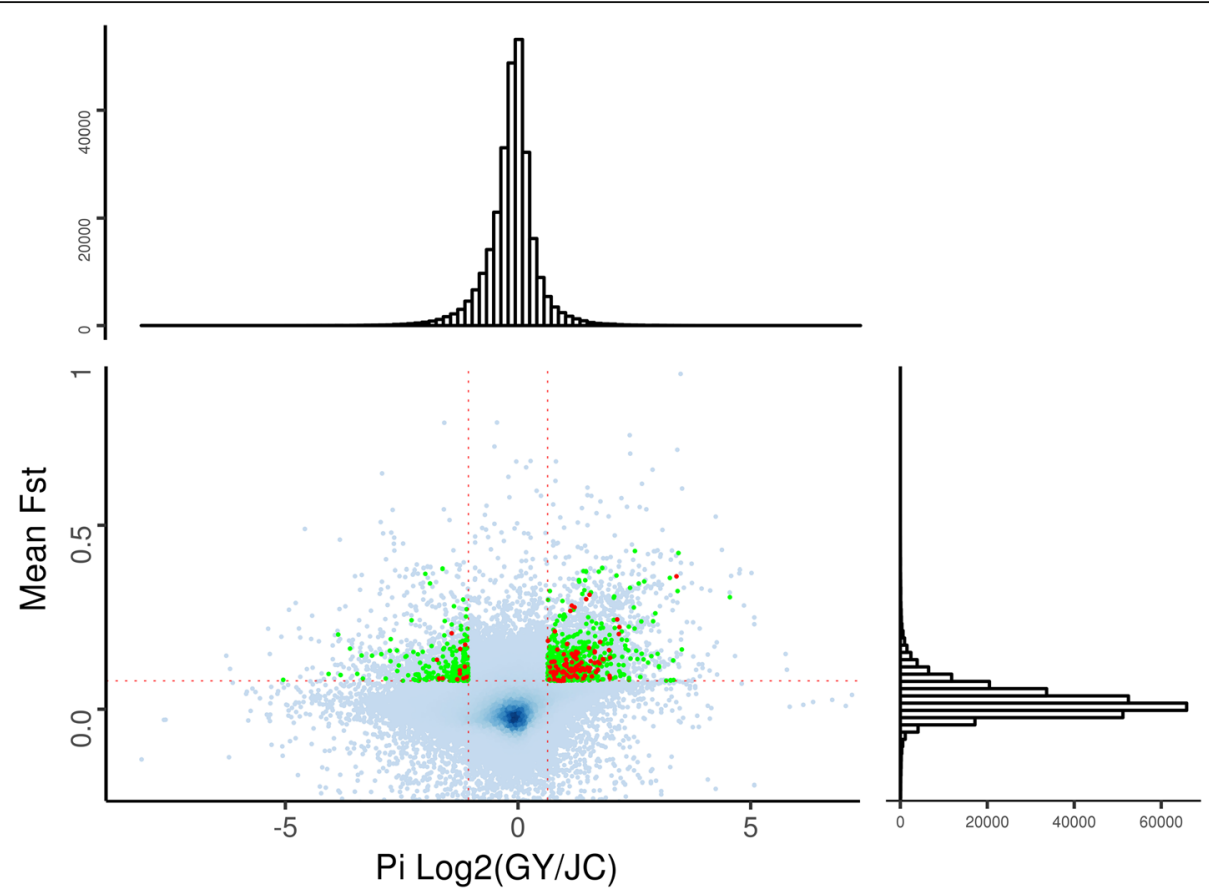

Fig. 5 Selective sweep analysis. In this plot, the darker the shade of the blue points, the greater the number of points present at that location. Horizontal and vertical red dotted lines are used to split the top 5\% of points from all other points. The points (windows) with the top 5\% Fst and $\log 2$ (pi ratio) simultaneously were colored green if they reside in a gene. Furthermore, the points were colored red if the genes they reside in were enriched in a biological terms. The upper and right histograms indicate the number of points in the corresponding intervals

Table 3 Enrichment analysis of the genes hit by the top $5 \%$ of points

\begin{tabular}{|c|c|c|}
\hline Population & Category & Enriched Term \\
\hline \multirow[t]{17}{*}{ JC (Valley yak) } & KEGG & Protein digestion and absorption \\
\hline & KEGG & Ether lipid metabolism \\
\hline & KEGG & alpha-Linolenic acid metabolism \\
\hline & KEGG & Glutamatergic synapse \\
\hline & KEGG & p53 signaling pathway \\
\hline & KEGG & Calcium signaling pathway \\
\hline & KEGG & Glycosaminoglycan biosynthesis - chondroitin sulfate / dermatan sulfate \\
\hline & KEGG & Retrograde endocannabinoid signaling \\
\hline & KEGG & Melanogenesis \\
\hline & KEGG & Pancreatic secretion \\
\hline & KEGG & Prolactin signaling pathway \\
\hline & KEGG & Circadian entrainment \\
\hline & GO_MF & calcium ion binding \\
\hline & GO_MF & NN-dimethylaniline monooxygenase activity \\
\hline & GO_CC & organelle membrane \\
\hline & GO_CC & extracellular region \\
\hline & GO_BP & lipid catabolic process \\
\hline \multirow[t]{4}{*}{ GY (Plateau yak) } & GO_MF & ligase activity \\
\hline & GO_MF & isomerase activity \\
\hline & KEGG & Adherens junction \\
\hline & KEGG & Tight junction \\
\hline
\end{tabular}


the other was related to domestication ('calcium signaling pathway', 'p53 signaling pathway', 'glutamatergic synapse' and 'retrograde endocannabinoid signaling'). For the GY population, only two pathways related to the cell junction were found to be enriched, which were adherens junctions and tight junctions. The regions under strong selective sweep were related to totally different functions in the two populations, which suggested these two populations underwent different adaptive evolution and it was consistent with their different geographical distribution.

\section{Marker-assisted selection Identify 330 candidate markers associated with the T15L5 trait}

The screening of loci associated with the T15L5 trait started from 498,542 loci harboring population-level genotypes (Fig. 1). Among them, 330 loci were selected as candidate markers for selective breeding because the populationlevel genotypes of these loci of the $\mathrm{YC}$ population were different from those of the ZC-GY population. A total of 61 genes harboring these loci or being within $5 \mathrm{~kb}$ of these loci were identified as possible causal genes of the T15L5 trait. The top 5 genes carrying the most loci were PPP2R2B (25 [harboring these loci] +0 [within $5 \mathrm{~kb}$ of these loci]), TBL1XR1 $(23+1)$, SHB $(10+2)$, TMEM192 $(0+9)$ and two genes (BPIFB3, $5+1$ and PCP4L1, $0+6$ ). According to the reports, PPP2R2B [21] and TBL1XR1 [22, 23] were most likely to be related to the T15L5 trait (the relationship between these two genes and the development of somites are depicted in the discussion section).

The selection of a specific trait is equivalent to the selection of the causal alleles/genotypes which manifest this trait. The alleles/genotypes that neighbor causal ones would also be selected together due to their associated linkage. Therefore, the number of candidate markers on each chromosome was determined and, as a result, the top 3 chromosomes carrying the most markers were chromosome 1 (39 loci, harboring TBL1XR1), chromosome 17 (35 loci, harboring TMEM192) and chromosome 7 (31 loci, harboring PPP2R2B) (Fig. 7). The genes carrying the most loci were also located on these three chromosomes, which indicated that QTLs determining the T15L5 trait of the Jinchuan yak were likely linked with or located within these genes.

A comparison of the sequences of the PPP2R2B gene from yak, cattle (Bos taurus) and goat (Capra hircus) uncovered a fact that the PPP2R2B gene sequence of yak was assembled into two contigs separately. The PPP2R2B genes from cattle and goat are comprised of eleven and ten exons respectively, and the total lengths are 509,525 bp and 478,425 bp. But the length of yak's PPP2R2B gene is $129,107 \mathrm{bp}$ which is made up of the last eight exons (including introns between these eight exons). The distance between cattle's first three exons (the head part, denoted by $\mathrm{H}$ ) and the last eight exons (the tail part, denoted by T) was $184,756 \mathrm{bp}$ and the total length of the last eight exons (including introns between these eight exons) was $128,197 \mathrm{bp}$. Moreover, the corresponding distances in goat were 188,301 bp (between first two exons $(\mathrm{H})$ and last eight exons $(\mathrm{T})$ ) and 130,901 bp (total length of the last eight exons including introns). The distant distance between $\mathrm{H}$ and $\mathrm{T}$ might be the reason for the loss of $\mathrm{H}$ in the yak, which may result from artificial annotation and not the intrinsic nature of this gene in yak. The similar lengths and sequences of the last eight exons between yak, cattle, and goat indicated the correctness and reliability of the annotated part of yak. No population-level variants were observed in the head part of yak's PPP2R2B gene. The reference genome of the latter two species has already reached the chromosome level but the reference genome of yak was still fragmented, which reminded us of the necessity of improving the yak's reference genome for further understanding the molecular mechanism underlying the yak's good characteristics.

\section{Definition and selection of best markers}

For developing markers used to select individuals with the T15L5 trait, a small portion of loci were further isolated from the 330 loci and validated in 51 extra samples. The three primary factors considered during selection were the haplotype diversity of a locus, the genes and the chromosomes where loci resided (Fig. 7) and the combination of alleles (genotype) of loci (heterozygous or homozygous). For the haplotype diversity, the genotypes of loci residing within $5 \mathrm{~kb}$ of 330 loci were extracted into 330 groups and each group of genotypes was phased with Beagle separately. For each locus, two haplotypes would be constructed. The haplotype diversity of a locus for a population was defined as the count of distinct haplotypes of this locus in this population. The haplotype diversity of each population is presented in Fig. 6. 25\% of loci represented 20 different haplotypes in the $\mathrm{YC}-\mathrm{ZC}$ population and should be avoided because of their high haplotype diversity. Another 25\% of loci representing no more than 14 different haplotypes were preferred due to their low haplotype diversity. The YC population was observed to have the highest haplotype diversity derived from these 330 loci because it had the largest median and the least loci with low haplotype diversity. It likely indicated that Jinchuan yaks with the T15L5 trait had relatively high genetic diversity in these loci.

For the genes and chromosomes (Fig. 7), those harboring the most loci were preferred so the loci that resided in the genes (PPP2R2B, TBL1XR1, and TMEM192) and the chromosomes (1, 7 and 17) had a high priority. For 


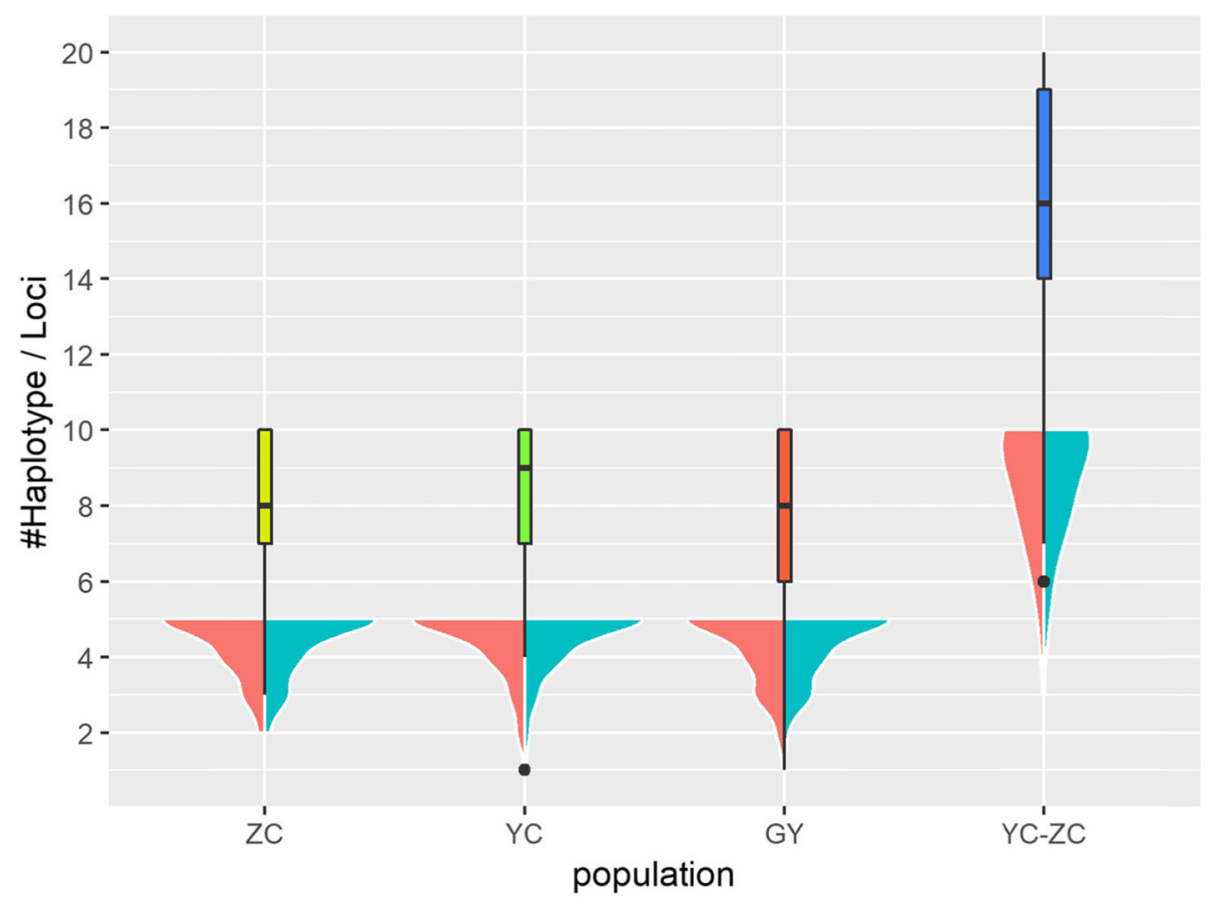

Fig. 6 The violin plot of the haplotype diversity. The boxplot of each population is shown according to the total number of haplotypes for each locus. The corresponding density plot of each population is composed of two parts. The two parts were asymmetry and separately represented the distribution of the number of the haplotypes of loci on one side

the combination of alleles of loci, the loci with homozygous genotypes for the YC population were preferred because the genotypes of progeny whose two parents had the same homozygous genotypes would be the same with their parents. The ratio of homozygous loci to heterozygous loci was 17:313. According to the criteria mentioned above, 13 loci were selected to be genotyped in 51 extra Jinchuan yaks with known thoracolumbar vertebral formula.

\section{7 best markers validated by target sequencing}

Considering the cost of further validation, a stepwise strategy was adopted to alleviate the bias introduced by population stratification. Ten loci were selected to be genotyped in 26 Jinchuan yaks (including the 15 resequenced samples used as the standard). According to the concordance between the genotypes of 15 resequenced samples obtained from the whole genome resequencing and the target sequencing, 3 loci were excluded. Therefore, 3 extra loci from the 330 candidate loci were chosen. The new ten loci were genotyped in 50 Jinchuan yaks (including the 10 resequencing samples). In summary, 51 extra Jinchuan yaks were genotyped with target sequencing and the evaluation of these 7 loci which appeared in two rounds of target sequencing is presented in Tables 4 and 5.

The evaluation consisted of two aspects: one was the ability to successfully predict 20 thoracolumbar vertebrae and the other was to predict 15 thoracic vertebrae. First, the loci whose major genotypes differed in two phenotype groups (e.g. the 'observed 19' group and the 'observed 20' group) were selected. The major genotypes denoted the genotype whose count was the most in one locus. Second, each individual was scored for two phenotypes according to all its selected genotypes and the rule of scoring was: +3 if the genotype probability $>=0.9 ;+2$ if the genotype probability $>=0.8$ and +1 for the rest. Third, if the difference of scores between two phenotypes (diff) was greater than a specific value, this individual was considered to be predictable using our loci and its phenotype was specified as the phenotype corresponding to the higher score. Others are specified as unknown. Finally, the efficiency of properly predicting a phenotype was assessed with 'accuracy of prediction' and 'representative capacity'. The accuracy of prediction was defined as the percentage of individuals with a correctly determined phenotype relative to the total individuals who were predicted to be this phenotype. The definition of representative capacity was the percentage of individuals with a correctly determined phenotype relative to the total individuals who were observed to be this phenotype.

In the evaluation of the ability of these 7 loci to predict 20 thoracolumbar vertebrae, 6 out of 51 individuals were expected to be 20 thoracolumbar vertebrae and 2 out of 6 were observed to be 20 thoracolumbar vertebrae. 


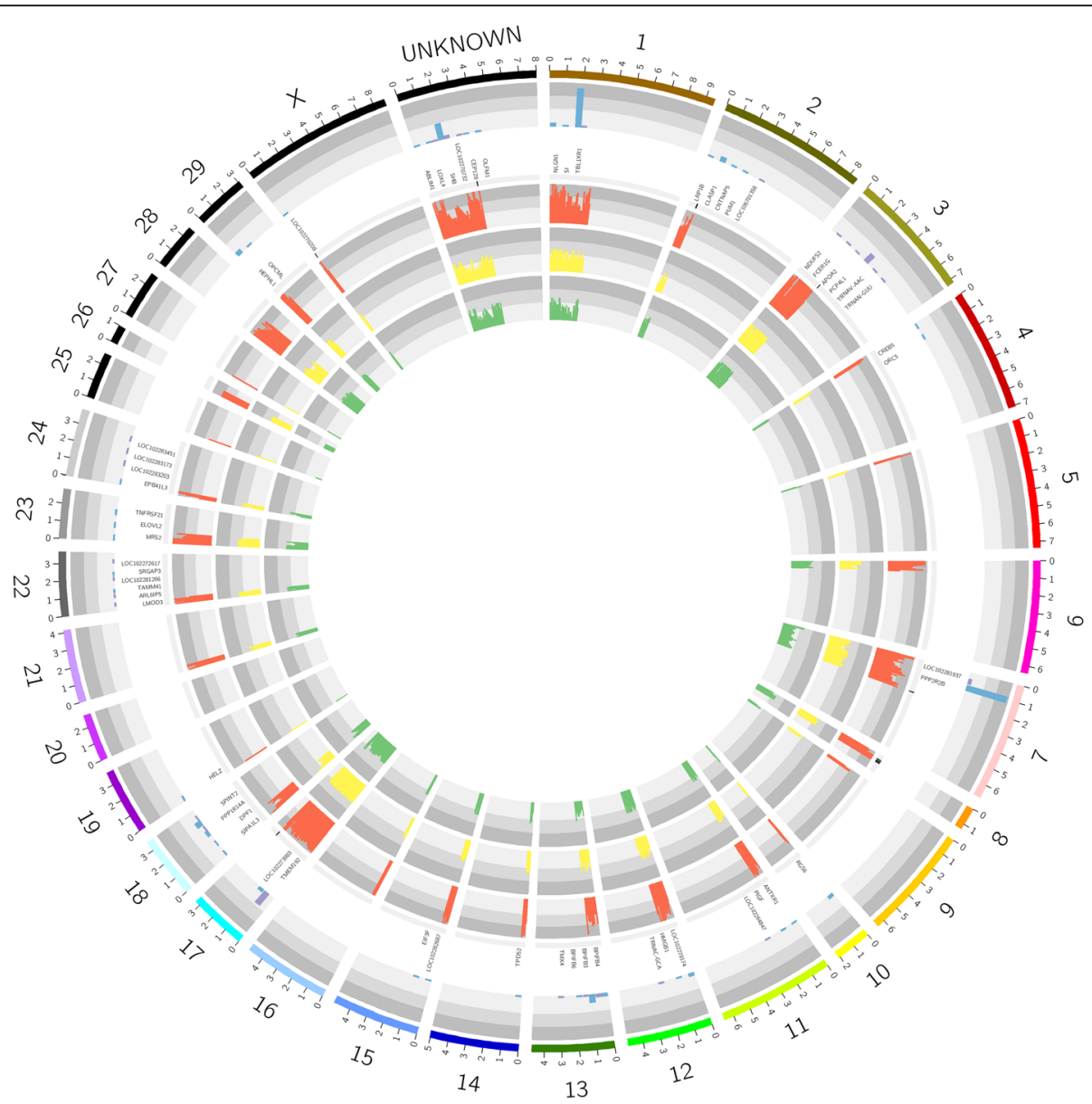

Fig. 7 Seven factors were considered in the selection of the 10 most reliable markers. Each factor corresponds to one of seven circles. A number from 1 to 7 was assigned to each circle from the outside to the inside. The first circle represents 29 autosomes, 1 X chromosome and 1 extra chromosome used to carry the contigs that were not assigned to chromosomes. The third circle is representative of the gene names; while the histogram shown in the second circle aligned with the third circle which represented the number of loci in this gene. Each gene corresponded to two bars: the left blue bar indicated the number of loci resided in this gene and the right purple bar indicated the loci located at the outside of the gene but within $5 \mathrm{~kb}$ of the gene. The 4th, 5th, 6th and 7th circles were aligned with each other and represented the conditions of each locus. The locus whose genotype was homozygous is shown as a black line in the fourth circle. The number of haplotypes for a locus was represented by the length of this locus' bar at the 5th, 6th, and 7th circle corresponding to the YC-ZC population, the YC population and the ZC population, respectively

Therefore, it indicated the accuracy of predicting 20 thoracolumbar vertebrae was $33.33 \%$. And 2 out of 10 individuals with 20 thoracolumbar vertebrae were identified, which indicated the representative capacity was $20 \%$. When it came to predicting 15 thoracic vertebrae, the accuracy increased to $100.00 \%$ but the representative capacity was still $20.00 \%$. One reason that could explain the difference between the accuracies of two conditions was the difference between 19 and 20 vertebrae was smaller than the difference between 14 and 15 thoracic vertebrae. The representative capacity told us the 10 Jinchuan yaks resequenced before only represented

Table 4 The evaluation of the ability of 7 loci predicting 20 vertebrae

\begin{tabular}{|c|c|c|c|c|}
\hline diff $>1$ & observed 19 & observed 20 & total & accuracy of prediction \\
\hline expected 19 & 24 & 4 & 28 & $85.71 \%$ \\
\hline expected 20 & 4 & 2 & 6 & $33.33 \%$ \\
\hline unknown & 13 & 4 & 17 & \\
\hline total & 41 & 10 & 51 & \\
\hline representative capacity & $58.54 \%$ & $20.00 \%$ & & \\
\hline
\end{tabular}


Table 5 The evaluation of the ability of 7 loci predicting 15 thoracic vertebrae

\begin{tabular}{llllr}
\hline diff $>4$ & observed 14 & observed 15 & total & accuracy of prediction \\
\hline expected 14 & 18 & 4 & 22 & $81.82 \%$ \\
expected 15 & 0 & 2 & 2 & $100.00 \%$ \\
unknown & 23 & 4 & 27 & 51 \\
total & 41 & 10 & & \\
representative capacity & $43.90 \%$ & $20.00 \%$ & & \\
\hline
\end{tabular}

around $20.00 \%$ of the total genetic diversity of individuals with T15L4/T15L5 traits.

\section{Discussion}

The objective of this study was to explore the genetic background of the Jinchuan yak and the possible mechanisms of aberrant somitogenesis according to the whole genome resequencing data in order to find markers which could be used to select individuals with the specific thoracolumbar vertebral formula (T15L5) trait. The representative capacity $(20.00 \%)$ was satisfying in our view considering our relatively small initial sample size and the use of only 7 markers to predict the trait. In the future, more loci could be included to increase the representative capacity of the genetic diversity at least in theory. After identifying the causal gene(s) or QTLs dominate for this trait, more precise selection could be carried out using the corresponding markers.
The predominant type of $\mathrm{PP} 2 \mathrm{~A}$ is a heterotrimer phosphatase, which consists of a scaffolding subunit A, a regulatory subunit $B$ and a catalytic subunit $C$ [24], and one of the substrates it could bind is APC [25], which is also the component of the beta-catenin degradation complex. Four gene families exist for the B subunit and the PPP2R2B gene identified in our study is in the PPP2R2 gene family [24]. PP2A complexes contain different regulatory $\mathrm{B}$ subunits that exist in disparate tissues at varying times [24]. PP2A is able to be recruited by the beta-catenin degradation complex carrying the component HSP105 to antagonize the phosphorylation of betacatenin and prevent beta-catenin from phosphorylationdependent ubiquitination, which leads to wnt-induced beta-catenin accumulation in the cytoplasm [26] (Fig. 8 a \& b). Msgn1 is reported to function as a direct target gene of Wnt3a performing the duty of transferring the spatial signal from the Wnt3a/beta-catenin gradient to the Notch
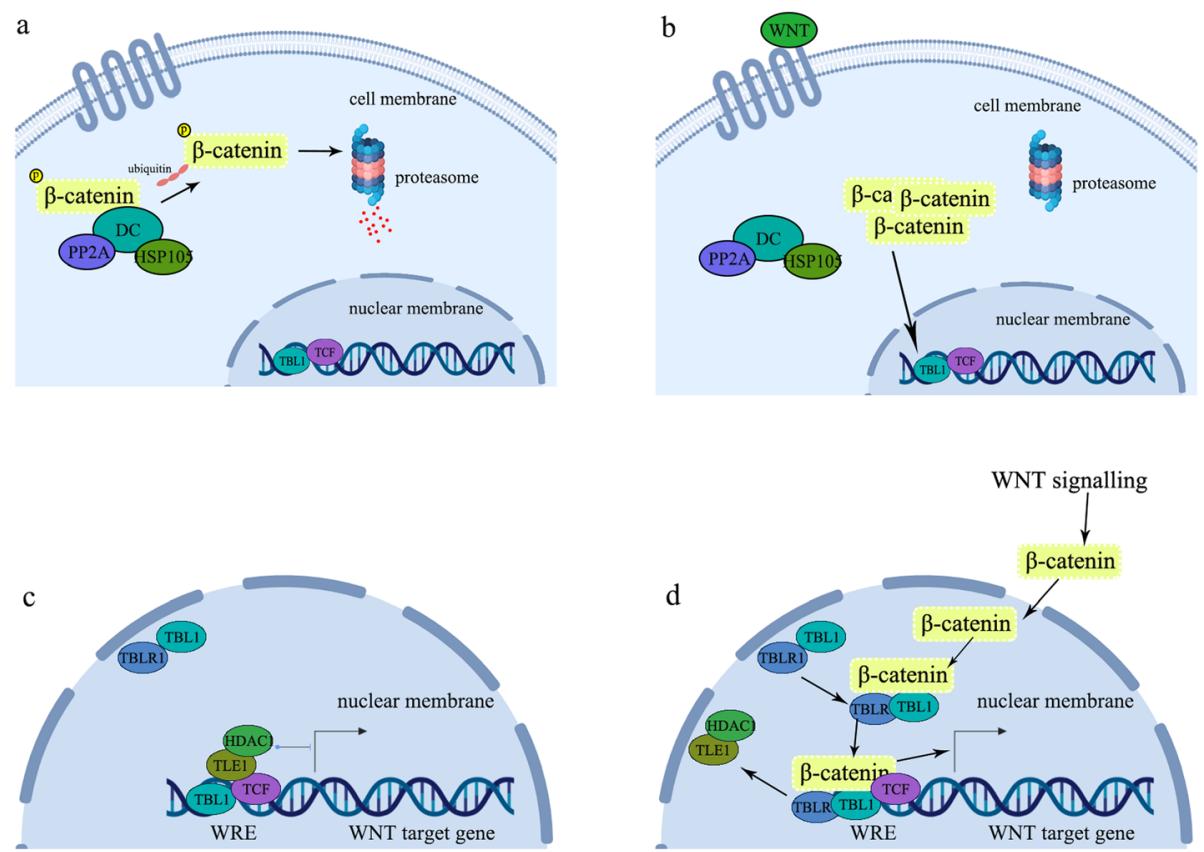

Fig. 8 Role of PP2A and TBLR1 in WNT/beta-catenin signaling pathway. a. beta-catenin is phosphorylated by the degradation complex (DC) with PP2A and HSP105 as its components, and it undergoes phosphorylation-dependent ubiquitination without the stimulus wnt and degraded by proteasome. $\mathbf{b}$. The accumulation of beta-catenin induced by the stimulus wnt. c. The co-repressors TLE1 and HDAC1 repress the wnt target genes. $\mathbf{d}$. TBL1-TBLR1 and beta-catenin recruit each to remove co-repressors TLE1 and HDAC1 and activate the transcription of wnt target genes. This figure is our own 
signaling program to control the segmentation clock during somitogenesis [21]. The period of the segmentation clock is regulated by intercellular coupling through the Notch signaling program and disruption of this coupling has been reported to increase the period of zebrafish somitogenesis and its embryonic somite length [27]. A faster segmentation clock and embryos with longer and shorter body segments were observed in transgenic zebrafish lines with elevated Notch signaling caused by a high copy number of DeltaD [7]. Altogether, the mutation of PPP2R2B gene was inferred to be the cause of the increased vertebral number of the Jinchuan yak. The variants of the PPP2R2B gene were primarily located in introns, similar to another study that also identified one genetic variant in the intron of the PPP2R2B gene and found it to be associated with altered breast cancer risk and recurrence [28].

Another important gene identified in this study that was found to carry numerous mutations was TBL1XR1 (also known as TBLR1), which was both involved in the signaling pathways regulating somite identity and somite number. The relationship between TBLR1 and somite identity originated from the corepressor SMRT [22]. TBLR1 is reported to be a core component of SMRT (silencing mediator of retinoic acid and thyroid hormone receptors) [23]. The establishment and maintainence of the somitic Hox code and segmental identity during embryonic development requires the SMRT-dependent repression of RAR (retinoic acid receptors) [22]. The ratio of thoracic to lumbar vertebrae changed from T13:L6 to T14:L5 in mice whose SMRT function in NR signaling was specifically disabled [22]. Therefore, the mutation of the TBLR1 gene might influence the normal function of SMRT and hence the segmental identity. As for the relationship between TBLR1 and the somite number, the necessity of mutual recruitment between TBLR1 and $\beta$-catenin for the activation of the transcription of the wnt3a target genes [29, 30] (Fig. $8 \mathrm{c}$ and d) indicated that the mutations in TBLR1 we observed here might influence the original function of $\beta$ catenin and hence the somite number as with the PPP2R2B gene.

When a species evolves under a certain pressure, the areas harboring alleles that help the species better adapt to this pressure would be under strong selective sweep. For example, the windows in which the SOCS2 and GPX3 genes reside were screened out with selective sweep analysis of Ovis aries based on sequencing data similar to ours. The functions of these genes did help the species to better adapt to their corresponding environments [31]. In our study, no genes were screened out under very strong selective sweep between Plateau yaks and Valley yaks. It might be explained by the fact that no extreme difference exists in the ecological environments between the Tibetan Plateau and the surrounding
Hengduan mountains because they are both at an altitude of at least $2000 \mathrm{~m}$ [1].

\section{Conclusions}

The increased vertebral number of yak in a natural condition is believed to be relevant to its adaption to plateau. Investigating the mechanism determining this trait and developing methods selecting such individuals would provide us better understanding of the mechanism regulating this trait and much more production yields. Our work provides a good practice of implementing MAS with the aid of modern NGS-based molecular methods to select yaks with one more thoracic vertebra. The report of the unique somitogenesis of yak and the discussion of possible mechanisms underlying this trait will inspire further exploration concerning the somitogenesis and plateau hypoxia. The interpretation of population genetics characteristics of this unique subpopulation of the yak builds a foundation for both selective breeding and evolutionary development. Also, the genomic resources of individuals whose thoracolumbar vertebral formula was confirmed by anatomical observation in our research provide researchers with a reliable foundation to carry out further comparative analysis.

\section{Methods}

\section{Grouping of samples and sequencing}

In order to explore the genetic characteristics of Jinchuan yaks with one additional thoracic vertebra, five Jinchuan yaks whose thoracolumbar vertebral formula was T15L5 (YC population) were taken as the case group and ten yaks with a normal vertebral number (five Jinchuan yaks (ZC population) and five Qinghai-plateau yaks (GY population) whose thoracolumbar vertebral formula was T14L5) were taken as the control group. Our samples could also be divided into another two groups according to a classical classification, which categorizes them as Plateau yaks (represented by the Qinghai-plateau yak) and Valley yaks (represented by the Jinchuan yak here; also named JC population) based on their geographic distribution and morphological features. Therefore the genetic characteristics at the population level of the Valley yak relative to the Plateau yak were also explored in this study. All study animals were slaughtered on breeding farms and the number of thoracic and lumbar vertebrae of each yak was determined to confirm its thoracolumbar vertebral formula. Ten Jinchuan yaks and five Qinghai-plateau yaks were from Jinchuan Yak Breeding Cooperative in Reta Village, Maori Township, Jinchuan county, Sichuan province and Qinghai Yushu Plateau Yak Farm in Yushu county, Qinghai province respectively. Whole blood was collected from each animal and used to extract genomic DNA. Pair-end libraries were constructed according to the protocol of the 
manufacturer (Illumina, San Diego, CA, USA) using the extracted genomic DNA. All samples were sequenced on an Illumina HiSeq X Ten sequencing platform.

\section{Reads alignment and variant calling}

The raw pair-end reads generated after sequencing were filtered out under the following conditions to generate clean reads: (a) It contained a sequencing adapter; (b) The length of ' $\mathrm{N}$ ' bases in either of a pair-end reads exceeded $10 \%$ of the total bases of the corresponding read; or (c) Low quality bases (Q-score $<=5$ ) in either of a pair-end reads that accounted for over $50 \%$ of total bases of the corresponding read. The clean reads were then mapped to the yak reference genome (BosGru_v2.0 [32]) with the BWA-MEM (v0.7.15) [33] using the parameter '-a'. The alignment results were further filtered using Picard (v2.5, http://broadinstitute.github.io/picard/ ) to remove duplicates with 'MarkDuplicates REMOVE DUPLICATES = true' and samtools [34] to remove records with 'view -F 3584 -q 30'. Variant calling was performed with the tools 'HaplotypeCaller' and 'GenotypeGVCF' in GATK (v3.6) [35]. ANNOVAR was used to annotate variants [36].

\section{Population genetic analysis}

The principle component analysis (PCA) was performed based on the whole genome SNVs of each individual using the program smartpca provided in EIGENSOFT (version 7.2.1) [37]. The squared correlated coefficient $\left(\mathrm{r}^{2}\right)$ of paired SNVs at certain distances was calculated using PopLDdecay [38] for each population. LD decay was visualized using ggplot2 according to $\mathrm{r}^{2}$ and its corresponding distance.

The pairwise (1-IBS) distance (IBS: identity-by-state) matrix between individuals was calculated with whole genome SNVs using PLINK [39] and used to construct a neighbor-joining (NJ) tree using PHYLIP [40]. The visualization of the tree was implemented by ggtree [41]. Cattle (Bos taurus) (NGS data accession ID: ERR035712 - ERR035769), whose whole genome SNVs were obtained according to the same method we used for our samples here, was selected as an outgroup to root the NJ tree.

Both the nucleotide diversity (pi) of the Jinchuan yak (composed of the $\mathrm{ZC}$ and the $\mathrm{YC}$ population) and Qinghai-plateau yak (the GY population) and the fixation index (following Weir and Cockerham's paper [42]) were calculated using vcftools [43] with a slidingwindow method $(50 \mathrm{~kb}$ windows and a $10 \mathrm{~kb}$ step length). The pi ratio (pi of GY/pi of JC) was log2transformed to generate a "log2 (pi ratio)". The enrichment analysis of the candidate genes that were covered by the windows with the top 5\% Fst and $\log 2$ (pi ratio) simultaneously were implemented with DAVID [44]. All yak annotated genes (BosGru_v2.0 [32]) were used as the background to displace the default background provided by DAVID.

\section{Marker-assisted selection}

The yak reference genome (BosGru_v2.0 [32]) we used in this study was aligned to a chromosomal level assembly of the yak reference genome (Accession ID: PRJNA435474. This chromosomal level assembly was obtained by scaffolding the reference genome we used here in virtue of the reference genome of closely related species cattle.) with LASTZ [45] to anchor scaffolds to their possible corresponding chromosomes. The genotypes of loci residing within $5 \mathrm{~kb}$ of the 330 loci were extracted into 330 groups and phased with Beagle [46] to help us evaluate the haplotype diversity of the region where each locus resided. Seven factors that we considered when we selected the most reliable candidate markers were illustrated with CIRCOS [47]. Thirteen loci out of 330 loci were picked out stepwise and target sequencing of these loci was carried out with Illumina NextSeq500. Primers used to amplify the sequences harboring the final seven loci are presented in S2 Table. All 51 extra Jinchuan yaks were from the same place as the ten Jinchuan yak. The reads generated here were mapped to the reference genome to call variants and generate the genotypes of these loci.

\section{Supplementary information}

Supplementary information accompanies this paper at https://doi.org/10. 1186/s12864-020-6598-9.

Additional file 1. The genes that overlapped with regions under strong selective sweeps.

Additional file 2. Primers used to amplify the sequences harboring the final seven loci.

\section{Abbreviations}

Fst: Population-differentiation statistic; GWAS: Genome-wide association study; IBS: Identity by state; kb: Kilo-base pair; LD: Linkage disequilibrium; m: Meter; MAS: Marker-assisted selection; NGS: Next-generation sequencing; NJ: Neighbor-joining; PC: Principal component; PCA: Principal component analysis; pi: Nucleotide diversity; PSM: Presomitic mesoderm;

QTL: Quantitative trait loci; RAR: Retinoic acid receptors; SMRT: Silencing mediator of retinoic acid and thyroid hormone receptors; SNP: Single nucleotide polymorphism; SNV: Single nucleotide variant; Tn1Ln2: The number of thoracic vertebrae is $\mathrm{n} 1$ and the number of lumbar vertebrae is $\mathrm{n} 2$; TVN: Thoracic vertebral number

\section{Acknowledgments}

Not Applicable.

Authors' contributions

YLW, MFJ, HYC, and XLL designed the research. YA and XLL collected the samples. YW, HYC MFJ, and YA analyzed the data. YW and MFJ wrote the paper. All authors have read and approved the manuscript.

\section{Funding}

This work was supported by a grant from National Key Technology Support Program (2014BAD13B03) to YLW, Sichuan Science and Technology Program 
(2019YFH0035) to MFJ and The National Natural Science Foundation of China (31172198) to MFJ. The funders had no role in the design of the study and collection, analysis, and interpretation of data and in writing the manuscript.

\section{Availability of data and materials}

The datasets generated and/or analysed during the current study are available in the NCBI repository (https://www.ncbi.nlm.nih.gov/) and are available under the accession number PRJNA516576.

\section{Ethics approval and consent to participate}

All methods were performed in accordance with the guidelines and regulations approved by The Animal Care and Use Committee of the Southwest Minzu University. Verbal consent was obtained from the breeding farms at which the slaughtered animals were sampled, which was sufficient to inform owners of animals and approved by the Animal Care and Use Committee of the Southwest Minzu University. All procedures and experiments were approved by the Animal Care and Use Committee of the Southwest Minzu University.

\section{Consent for publication}

Not applicable.

\section{Competing interests}

The authors declare that they have no competing interests.

\section{Author details}

${ }^{1}$ College of Life Science and Technology, Southwest Minzu University, Chengdu 610041, Sichuan, China. ${ }^{2}$ Center of Growth, Metabolism, and Aging, Key Laboratory of Bio-Resources and Eco-Environment, College of Life Sciences, Sichuan University, Chengdu 610064, Sichuan, China. ${ }^{3}$ Sichuan Academy of Grassland Sciences, Chengdu, Sichuan, China. ${ }^{4}$ Key Laboratory of Sichuan Province for Qinghai-Tibetan Plateau Animal Genetic Resource Reservation and Exploitation, Chengdu 610041, China.

\section{Received: 27 October 2019 Accepted: 19 February 2020}

Published online: 04 March 2020

\section{References}

1. Mipam TD, Wen Y, Fu C, Li S, Zhao H, Ai Y, et al. Maternal phylogeny of a newly-found yak population in China. Int J Mol Sci. 2012;13(9):11455-70.

2. Li Q, Fu C, Wen Y, Li J, Luo G, Li S. Determination and correlation analysis of body size and slaughter performance of Jinchuan multi-ribbed yak. China Herbivore Sci. 2012;32(02):18-20.

3. Yang J, Wen Y, Feng Z, Ke M, Gao X, An D. Correlation analysis for beef performance and multi-vertebra properties of Jinchuan yak. J Domestic Animal Ecol. 2015;36(09):26-30.

4. Hirsinger $E$, Jouve C, Dubrulle J, Pourquie O. Somite formation and patterning. Int Rev Cytol. 2000;198:1-65.

5. Gomez C, Ozbudak EM, Wunderlich J, Baumann D, Lewis J, Pourquie O. Control of segment number in vertebrate embryos. Nature. 2008;454(7202): 335-9.

6. Carapuco M, Novoa A, Bobola N, Mallo M. Hox genes specify vertebral types in the presomitic mesoderm. Genes Dev. 2005;19(18):2116-21.

7. Liao B-K, Jörg DJ, Oates AC. Faster embryonic segmentation through elevated Delta-notch signalling. Nat Commun. 2016;7:11861.

8. Scaal M. Early development of the vertebral column. Semin Cell Dev Biol. 2016:49:83-91.

9. Han H, Wang S, Huang J, Chen H, Lei C. Investigation of Multi-vertebral Trait in Qinchuan Cattle. China Cattle Sci. 2013;39(06):78-79;81.

10. Li S, Luo R, Lai D, Ma M, Hao F, Qi X, et al. Whole-genome resequencing of Ujumqin sheep to investigate the determinants of the multi-vertebral trait. Genome. 2018;61(9):653-61.

11. Fan $Y$, Xing $Y$, Zhang Z, Ai H, Ouyang Z, Ouyang J, et al. A further look at porcine chromosome 7 reveals VRTN variants associated with vertebral number in Chinese and Western pigs. PLoS One. 2013;8(4):e62534.

12. Rohrer GA, Nonneman DJ, Wiedmann RT, Schneider JF. A study of vertebra number in pigs confirms the association of vertnin and reveals additional QTL. BMC Genet. 2015;16:129.
13. Zhang L, Liu X, Liang J, Zhao K, Yan H, Li N, Pu L, Zhang Y, Shi H, Wang L. Genome-wide association study for number of vertebrae in an f2 large whitex minzhu population of pigs. BioRxiv. 2015:016956.

14. Shaw A. Variations in the skeletal structure of the pig. Sci Agric. 1929;10(1): 23-7.

15. Ren DR, Ren J, Ruan GF, Guo YM, Wu LH, Yang GC, et al. Mapping and fine mapping of quantitative trait loci for the number of vertebrae in a White Duroc $\times$ Chinese Erhualian intercross resource population. Anim Genet. 2012:43(5):545-51.

16. Mikawa S, Sato S, Nii M, Morozumi T, Yoshioka G, Imaeda N, et al. Identification of a second gene associated with variation in vertebral number in domestic pigs. BMC Genet. 2011;12:5.

17. Duan $Y$, Zhang $H$, Zhang Z, Gao J, Yang J, Wu Z, et al. VRTN is required for the development of thoracic vertebrae in mammals. Int J Biol Sci. 2018; 14(6):667.

18. Li S, Wen Y, Ma D, Yang J, Hou D, Li Q, et al. The population survey of Jinchuan multi-rib yak. Acta Ecologiae Animalis Domastici. 2014;35(1):65-70.

19. Wang Z, Shen X, Liu B, Su J, Yonezawa T, Yu Y, et al. Phylogeographical analyses of domestic and wild yaks based on mitochondrial DNA: new data and reappraisal. J Biogeogr. 2010;37(12):2332-44.

20. Qiu Q, Wang L, Wang K, Yang Y, Ma T, Wang Z, et al. Yak whole-genome resequencing reveals domestication signatures and prehistoric population expansions. Nat Commun. 2015;6:10283.

21. Chalamalasetty RB, Dunty WC Jr, Biris KK, Ajima R, lacovino M, Beisaw A, et al. The Wnt3a/beta-catenin target gene Mesogenin1 controls the segmentation clock by activating a notch signalling program. Nat Commun. 2011;2:390.

22. Hong SH, Fang S, Lu BC, Nofsinger R, Kawakami Y, Castro GL, et al. Corepressor SMRT is required to maintain Hox transcriptional memory during somitogenesis. Proc Natl Acad Sci U S A. 2018;115(41):10381-6.

23. Li JY, Daniels G, Wang J, Zhang X. TBL1XR1 in physiological and pathological states. Am J Clin Exp Urol. 2015;3(1):13-23.

24. Virshup DM, Shenolikar S. From promiscuity to precision: protein phosphatases get a makeover. Mol Cell. 2009;33(5):537-45.

25. Seeling JM, Miller JR, Gil R, Moon RT, White R, Virshup DM. Regulation of beta-catenin signaling by the B56 subunit of protein phosphatase $2 \mathrm{~A}$. Science. 1999;283(5410):2089-91.

26. Yu N, Kakunda M, Pham V, Lill JR, Du P, Wongchenko M, et al. HSP105 recruits protein phosphatase $2 \mathrm{~A}$ to dephosphorylate beta-catenin. Mol Cell Biol. 2015;35(8):1390-400.

27. Herrgen L, Ares S, Morelli LG, Schroter C, Julicher F, Oates AC. Intercellular coupling regulates the period of the segmentation clock. Curr Biol. 2010; 20(14):1244-53.

28. Vazquez A, Kulkarni D, Grochola LF, Bond GL, Barnard N, Toppmeyer D, et al A genetic variant in a PP2A regulatory subunit encoded by the PPP2R2B gene associates with altered breast cancer risk and recurrence. Int J Cancer. 2011;128(10):2335-43.

29. Li J, Wang CY. TBL1-TBLR1 and beta-catenin recruit each other to Wnt target-gene promoter for transcription activation and oncogenesis. Nat Cell Biol. 2008;10(2):160-9.

30. Choi HK, Choi KC, Yoo JY, Song M, Ko SJ, Kim CH, et al. Reversible SUMOylation of TBL1-TBLR1 regulates beta-catenin-mediated Wnt signaling. Mol Cell. 2011:43(2):203-16.

31. Yang J, Li WR, Lv FH, He SG, Tian SL, Peng WF, et al. Whole-genome sequencing of native sheep provides insights into rapid adaptations to extreme environments. Mol Biol Evol. 2016;33(10):2576-92.

32. Qiu Q, Zhang G, Ma T, Qian W, Wang J, Ye Z, et al. The yak genome and adaptation to life at high altitude. Nat Genet. 2012;44(8):946-9.

33. Li H, Durbin R. Fast and accurate short read alignment with burrowswheeler transform. Bioinformatics. 2009;25(14):1754-60.

34. Li H, Handsaker B, Wysoker A, Fennell T, Ruan J, Homer N, et al. The sequence alignment/map format and SAMtools. Bioinformatics. 2009;25(16):2078-9.

35. McKenna A, Hanna M, Banks E, Sivachenko A, Cibulskis K, Kernytsky A, et al. The genome analysis toolkit: a MapReduce framework for analyzing nextgeneration DNA sequencing data. Genome Res. 2010;20(9):1297-303.

36. Wang $\mathrm{K}$, Li M, Hakonarson $\mathrm{H}$. ANNOVAR: functional annotation of genetic variants from high-throughput sequencing data. Nucleic Acids Res. 2010; 38(16):e164.

37. Price AL, Patterson NJ, Plenge RM, Weinblatt ME, Shadick NA, Reich D. Principal components analysis corrects for stratification in genome-wide association studies. Nat Genet. 2006;38(8):904 
38. Zhang C, Dong S-S, Xu J-Y, He W-M, Yang T-L. PopLDdecay: a fast and effective tool for linkage disequilibrium decay analysis based on variant call format files. Bioinformatics. 2018;35(10):1786-8.

39. Chang CC, Chow CC, Tellier LC, Vattikuti S, Purcell SM, Lee JJ. Secondgeneration PLINK: rising to the challenge of larger and richer datasets. Gigascience. 2015:4:7.

40. Felsenstein J. PHYLIP-phylogeny inference package (version 3.2). Cladistics. 1989:5:164-6.

41. Yu G, Smith DK, Zhu H, Guan Y, Lam TTY. Ggtree: an R package for visualization and annotation of phylogenetic trees with their covariates and other associated data. Methods Ecol Evol. 2017;8(1):28-36.

42. Weir BS, Cockerham CC. Estimating F-statistics for the analysis of population structure. Evolution; Int J Organic Evolution. 1984;38(6):1358-70

43. Danecek P, Auton A, Abecasis G, Albers CA, Banks E, DePristo MA, et al. The variant call format and VCFtools. Bioinformatics. 2011;27(15):2156-8.

44. Huang da W, Sherman BT, Lempicki RA. Systematic and integrative analysis of large gene lists using DAVID bioinformatics resources. Nat Protoc. 2009; 4(1):44-57.

45. Harris RS. Improved pairwise Alignmnet of genomic DNA. Pennsylvania State University: Doctoral dissertation; 2007.

46. Browning SR, Browning BL. Rapid and accurate haplotype phasing and missing-data inference for whole-genome association studies by use of localized haplotype clustering. Am J Hum Genet. 2007;81(5):1084-97.

47. Krzywinski M, Schein J, Birol I, Connors J, Gascoyne R, Horsman D, et al. Circos: an information aesthetic for comparative genomics. Genome Res. 2009;19(9):1639-45.

\section{Publisher's Note}

Springer Nature remains neutral with regard to jurisdictional claims in published maps and institutional affiliations.

Ready to submit your research? Choose BMC and benefit from:

- fast, convenient online submission

- thorough peer review by experienced researchers in your field

- rapid publication on acceptance

- support for research data, including large and complex data types

- gold Open Access which fosters wider collaboration and increased citations

- maximum visibility for your research: over $100 \mathrm{M}$ website views per year

At $\mathrm{BMC}$, research is always in progress.

Learn more biomedcentral.com/submissions 